Noname manuscript No.

(will be inserted by the editor)

\title{
Polynomial (chaos) approximation of maximum eigenvalue functions
}

Efficiency and limitations

Luca Fenzi · Wim Michiels

\begin{abstract}
This paper is concerned with polynomial approximations of the spectral abscissa function (defined by the supremum of the real parts of the eigenvalues) of a parameterized eigenvalue problem, which are closely related to polynomial chaos approximations if the parameters correspond to realizations of random variables. Unlike previous work, we highlight the major role of this function smoothness properties. Even if the eigenvalue problem matrices are analytic functions of the parameters, the spectral abscissa function may not be differentiable, and even non-Lipschitz continuous, due to multiple rightmost eigenvalues counted with multiplicity. This analysis demonstrates smoothness properties not only heavily affect the approximation errors of the Galerkin and collocation based polynomial approximations, but also the numerical errors in the evaluation of coefficients in the Galerkin approach with integration methods. A documentation of the experiments, conducted on the benchmark problems through the software Chebfun, is publicly available.
\end{abstract}

Keywords Polynomial approximation · Polynomial chaos · Eigenvalue analysis · Interpolation · Integration methods

\section{Introduction}

Polynomial approximation is a pillar of approximation theory and strongly connected with the Polynomial Chaos (PC) method used in uncertainty quan-

L. Fenzi

Department of Computer Science, NUMA Section, KU Leuven, Belgium

Tel.: +3216376477

E-mail: Luca.Fenzi@cs.kuleuven.be

ORCID: 0000-0003-2840-3816

W. Michiels

Department of Computer Science, NUMA Section, KU Leuven, Belgium

Tel.: +3216327064

E-mail: Wim.Michiels@cs.kuleuven.be

ORCID: 0000-0002-0877-0080 
tification; as described in [36] and [19, 45, 20], respectively. The PC method is often used to approximate a quantity of interest as a function of uncertain parameters and to then extract relevant statistical information from the approximation. PC expansion of eigenvalue functions has recently attracted significant research focus: refer to $[11,26,30,7]$ for the standard eigenvalue problem and [38] for eigenvalue problems associated to delay differential equations.

Polynomial approximation accuracy crucially relies on the smoothness property of the function to be approximated. The aim of this work is to systematically show how the different behaviors of rightmost or dominant eigenvalues, particularly spectral abscissa function, can affect the polynomial (or PC) based approximations as well as numerical computation of coefficients; still the results on smoothness properties, here presented, are valid for polynomial (chaos) approximation of general real functions. The smoothness properties of eigenvalue function is rarely taken into account in the uncertainty quantification literature, see e.g. $[13,17,11,42,26,30,7,38]$ and reference therein.

The analysis focus on eigenvalue problems inferred from a linear autonomous system of delay differential equations,

$$
\left(\lambda I_{n}-\sum_{i=0}^{h} A_{i}(\omega) e^{-\lambda \tau_{i}(\omega)}\right) v=0, \lambda \in \mathbb{C}, v \in \mathbb{C}^{n} \backslash\{0\},
$$

where $I_{n}$ is the identity matrix of dimension $n, \omega \in \mathbb{S} \subset \mathbb{R}^{D}$ models parameters subject to uncertainty, $h, D \in \mathbb{N}$, and for every $i \in\{0, \ldots, h\}, A_{i}: \mathbb{S} \mapsto \mathbb{R}^{n \times n}$ and $\tau_{i}: \mathbb{S} \mapsto \mathbb{R}_{>0}$ are smooth functions. The eigenvalue problem (1) plays an important role in the stability analysis. For instance, the time delay system associated to (1) given $\omega$,

$$
\dot{x}(t)=\sum_{i=0}^{h} A_{i}(\omega) x\left(t-\tau_{i}(\omega)\right),
$$

is asymptotically stable if and only if the real part of the rightmost eigenvalue is negative, since the number of eigenvalues in each right half plane is finite [22]; or equivalently, if and only if the spectral abscissa

$$
\alpha(\omega)=\max _{\lambda \in \mathbb{C}}\left\{\Re(\lambda): \operatorname{det}\left(\lambda I_{n}-\sum_{i=0}^{h} A_{i}(\omega) e^{-\lambda \tau_{i}(\omega)}\right)=0\right\}
$$

is negative.

As a motivation for considering the delay eigenvalue problem, the smoothness properties of the spectral abscissa function represents a major concern in control design of time delay systems. The spectra of these systems features an infinite number of eigenvalues; therefore a implementable finite-dimensional controller is under-actuated, and its design in the frequency domain often reduces to shaping the overall spectrum with a finite number of degree of freedom. The state-of-art methods for stabilizing an unstable system or for 
improving the decay rate of solutions to equilibrium is based on minimizing the spectral abscissa as a function of controller parameter [23]. This procedure favors situations where the spectral abscissa is not smooth, since minima of the spectral abscissa are often characterized by defective rightmost eigenvalues. Moreover, several analytic control design techniques based on assigning directly multiple eigenvalues have been recently considered [28, 27, 40, 18, 3, 4]. These design techniques are simple but they provide, in general, non-smooth behaviors of the spectral abscissa for the controlled system, yet in the corresponding literature no attention is paid to robustness against parameter variations, while the use of standard analysis tools like the polynomial chaos expansion may become questionable.

Let $\left\{p_{i}(\omega)\right\}_{i=0}^{\infty}$ be a degree graded polynomial basis orthogonal w.r.t. a smooth non-negative function $\rho(\omega)$ defined and normalized on the compact support $\mathbb{S} \subset \mathbb{R}^{D}$, i.e. $\int_{\mathbb{S}} \rho(\omega) \mathrm{d} \omega=1$. The spectral abscissa function

$$
\alpha: \mathbb{S} \subset \mathbb{R}^{D} \rightarrow \mathbb{R}, \omega \mapsto \alpha(\omega),
$$

presents a polynomial expansion in the basis $\left\{p_{i}(\omega)\right\}_{i=0}^{\infty}$

$$
\alpha(\omega)=\sum_{i=0}^{\infty} c_{i} p_{i}(\omega),
$$

where the coefficients $c_{i}$ are evaluated by

$$
c_{i}=\frac{\left\langle\alpha, p_{i}\right\rangle_{\rho}}{\left\langle p_{i}, p_{i}\right\rangle_{\rho}}, \quad i \in \mathbb{N}
$$

The $\rho$-inner product, used in the above equation, is defined for all $f, g: \mathbb{S} \rightarrow \mathbb{R}$ such that

$$
\langle f, g\rangle_{\rho}=\int_{\mathbb{S}} f(\omega) g(\omega) \rho(\omega) \mathrm{d} \omega .
$$

and the induced $\rho$-norm is determined by $\|f\|_{\rho}=\sqrt{\langle f, f\rangle_{\rho}}$.

If $\omega$ is considered as a realization of a real random vector $\boldsymbol{\omega}$, with probability density function $\rho(\omega)$, then $\alpha(\boldsymbol{\omega})$ is also a random variable and its polynomial expansion

$$
\alpha(\boldsymbol{\omega})=\sum_{i=0}^{\infty} c_{i} p_{i}(\boldsymbol{\omega}),
$$

corresponds to a PC expansion of the spectral abscissa function with germ $\boldsymbol{\omega}$.

The PC expansion permits to compactly define a general random variable, through the chaos coefficients $c_{i}$, and the germ $\boldsymbol{\omega}$, which specifies also the $\rho$-orthonormal polynomial basis $\left\{p_{i}\right\}_{i}$. The chaos coefficients $c_{i}$ convey mean, variance and variance-based sensitivity analysis of the random variable. Indeed, other than for the computation of the mean and variance, they permit to decompose the variance into the contribution of individual model parameter and the interaction between each of them (Sobol's sensitivity index) or to measure the total variance due to each single model parameter (total order sensitivity index). We refer to $[14,32]$ for further information on sensitivity indexes. 
Theorem 1 Given PC expansion (8), the following formulas hold: ${ }^{1}$

1. Mean: $\mathbb{E}(\alpha(\boldsymbol{\omega}))=c_{0}$.

2. Variance: $\operatorname{Var}(\alpha(\boldsymbol{\omega}))=\sum_{i=1}^{\infty} c_{i}^{2}\left\langle p_{i}, p_{i}\right\rangle_{\rho}$.

3. If the polynomial basis $\left\{p_{i}(\omega)\right\}_{i=0}^{\infty}$ is constructed by tensor product of univariate polynomial bases $\left\{p_{i, d}\left(\omega_{d}\right)\right\}_{d=1, \ldots, D}^{i=0, \ldots, \infty}$ through the bijective D-tupling function $\varphi_{D}: \mathbb{N}^{D} \rightarrow \mathbb{N},\left(i_{1}, \ldots, i_{D}\right) \mapsto i$ in such a way that

$$
p_{i}(\omega)=\prod_{d=1}^{D} p_{i_{d}, d}\left(\omega_{d}\right), \quad\left(i_{1}, \ldots, i_{D}\right)=\varphi_{D}^{-1}(i),
$$

then, the Sobol's sensitivity index $\mathcal{S}_{\mathfrak{h}}(\alpha(\boldsymbol{\omega}))$ associated to the subset of uncertain parameters $\boldsymbol{\omega}_{\mathfrak{h}}$, where $\mathfrak{h}=\left(h_{1}, \ldots, h_{\ell}\right)$ is an element of the power set of $\{1, \ldots, D\}$ except the empty set, is determined by

$$
\mathcal{S}_{\mathfrak{h}}(\alpha(\boldsymbol{\omega}))=\sum_{i \in Q_{\mathfrak{h}}} \frac{c_{i}^{2}\left\langle p_{i}, p_{i}\right\rangle_{\rho}}{\operatorname{Var}(\alpha(\boldsymbol{\omega}))},
$$

where $Q_{\mathfrak{h}}=\left\{i \in \mathbb{N}: \varphi_{D}^{-1}(i)=\left(i_{h_{1}}, \ldots, i_{h_{D}}\right)\right.$, and $\left(i_{h_{1}}, \ldots, i_{h_{\ell}}\right)>$ $\left.0,\left(i_{h_{\ell+1}}, \ldots, i_{h_{D}}\right)=0\right\}$. For $d \in\{0, \ldots, D\}$, the effect of the dth parameter $\omega_{d}$ can be quantified by the total order sensitivity index

$$
\mathcal{S}_{d}(\alpha(\boldsymbol{\omega}))=\sum_{\substack{\mathfrak{h}=\left(h_{1}, \ldots, h_{\ell}\right), \exists 1 \leq j \leq \ell}} \mathcal{S}_{\mathfrak{h}}(\alpha(\boldsymbol{\omega})) .
$$

The choice of the polynomial basis $\left\{p_{i}\right\}_{i}$ differentiates the PC approach from polynomial approximation. Indeed, in the former the basis is orthonormal w.r.t. probability density function $\rho(\omega)$ of the random vector $\boldsymbol{\omega}$, in order to apply Theorem 1; for example, the Legendre polynomials are orthonormal to the probability density functions of uniform distributions [45, 19]. On the contrary, the latter approach ground the choice of the polynomial basis on existing results and on available numerical methods [36].

The present work highlights the fundamental role of the behavior of the spectral abscissa function $\alpha(\omega)$ in (4) w.r.t. truncations of its polynomial expansion (5) and the corresponding PC expansion (8) of $\alpha(\boldsymbol{\omega})$. In addition, this paper explicits the parallelism between PC theory and polynomial approximation, applying the results of the latter into the former context. To this end, the asymptotic convergence rate of polynomial (chaos) approximation are stated in $L^{\infty}$ norm, which is rarely employed in the context of PC theory, even though it is more stringent than $L^{2}$ and $L^{1}$.

The paper is organized as follows. In Section 2, we briefly review the behaviors of spectral abscissa functions furnishing text examples which are going to be analyzed in Section 3, where the polynomial approximation for $D=1$ parametric eigenvalue problems is investigated. Then, in Section 4, the polynomial

\footnotetext{
1 A proof of the first two classical results 1 and 2 can be found in [38], while the last result 3 is derived by [6].
} 
approximation for $D>1$ is analyzed through some numerical experiments. Finally, the text concludes in Section 5 with a summary of the novel findings as well as perspectives on future research directions. A MATLAB tutorial with the numerical experiments here presented can be found in [9].

\section{Smoothness property of the spectral abscissa function}

Due to the link between PC expansion (8) of $\alpha(\boldsymbol{\omega})$ and the polynomial approximation of spectral abscissa function (4), the smoothness properties of this function play a fundamental role in the analysis. In this section, we characterize the different behaviors of the spectral abscissa furnishing benchmark examples, which are analyzed in the following Section 3 . The reader is referred to $[16,8,25]$ for relevant results on the behavior of spectral abscissa functions.

The different behaviors of the spectral abscissa can be inferred by the analysis of the active eigenvalues, which are eigenvalues, $\lambda$ in (1), with real part equal to the spectral abscissa, $\Re(\lambda)=\alpha$, and non-negative imaginary part, $\Im(\lambda) \geq 0$.

The spectral abscissa is a continuous function which is smooth almost everywhere in $\mathbb{S}$, however in a set of measure zero, it may not be differentiable due to the presence of multiple active eigenvalues (counted with multiplicity).

SAE - Simple Active Eigenvalue. For all $\omega \in \mathbb{S}$, there is only one active eigenvalue, whose algebraic and geometric multiplicity is equal to 1 . The spectral abscissa is smooth over all the uncertain domain $\mathbb{S}$, i.e. $\alpha \in \mathcal{C}^{\infty}(\mathbb{S}, \mathbb{R})$.

MSSAEs - Multiple (Semi)-Simple Active Eigenvalues. For some $\omega \in \mathbb{S}$, there is more than one active eigenvalue. If the multiplicity of an active eigenvalue is greater than one, then it is semi-simple (i.e. the algebraic multiplicity is equal to the geometric one). In this case, the spectral abscissa may not be everywhere differentiable, however it is Lipschitz continuous in $\mathbb{S}$.

MNSSAEs - Multiple Non-Semi-Simple Active Eigenvalues. There exist $\omega \in \mathbb{S}$, where the algebraic multiplicity of an active eigenvalue is greater than the geometric one (i.e. it is non-semi-simple). In this case, the spectral abscissa is, in general, not even everywhere locally Lipschitz continuous.

The non-smooth behaviors often occur in the stabilization of time delay systems. Indeed, an eigenvalue-based approach to stabilize a infinite dimensional eigenvalue problem designs a finite controller, which minimizes the active eigenvalue [8]. This minimization leads to multiple (and, in general, nonsemi-simple) active eigenvalues, whose multiplicity is mainly equal to the dimension of the controller plus one. This situation occurs in Example 2, in the upcoming Section 4. Additional novel control design technique directly impose multiple non-semi-simple active eigenvalues in the spectrum of the controlled time delay system $[27,40,18,28,3,4]$.

Example 1 The characterization of the behaviors of the spectral abscissa function can be illustrated by the following parametric eigenvalue problems, where 
$\omega \in[-1,1]$

$$
\begin{aligned}
\left(\lambda I_{2}-\left(\begin{array}{cc}
e^{\omega} & 0 \\
0 & -1
\end{array}\right)\right) v & =0 ; \\
\left(\lambda I_{2}-\left(\begin{array}{cc}
\omega & 0 \\
0 & 0
\end{array}\right)\right) v & =0 ; \\
\left(\lambda I_{2}-\left(\begin{array}{cc}
0 & \omega \\
1 & 0
\end{array}\right)\right) v & =0 .
\end{aligned}
$$

The real parts of the spectra are shown in Figure 1. The first eigenvalue problem, (SAE), presents a smooth spectral abscissa function, the second one, (MSSAEs), presents a piecewise linear function (for $\omega=0$ the active eigenvalue is multiple semi-simple), while the last one, (MNSSAEs), shows the splitting of a double eigenvalue at $\omega=0$ with a square root behavior for $\omega \in[0,1]$.
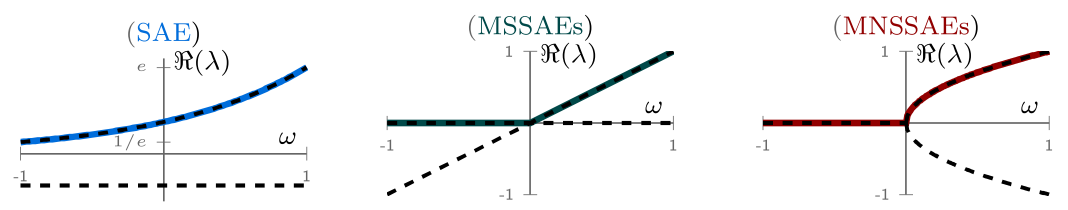

Fig. 1: Real parts of the spectra varying the $\omega$ parameter in $[-1,1]$ of the eigenvalue problems defined in Example 1. The spectral abscissa functions are highlighted.

Even though SAE, MSSAEs, and MNSSAEs cases are mutually exclusive, close to MNSSAEs cases the Lipschitz constant of the spectral abscissa might be very large. This phenomenon might have a significant effect on the transient behavior of the errors as a function of the approximation degree, but it does not affect the asymptotic convergence behavior of the polynomial approximation. For simplicity, this situation is not investigated in this text, since we focus on the asymptotic convergence rate of the polynomial (chaos) approximation.

\section{Analysis of the polynomial approximation for $D=1$}

In practice, for computational reasons, polynomial expansion (5) is approximated by finitely many terms

$$
\alpha_{P}(\omega)=\sum_{i=0}^{P} \tilde{c}_{i} p_{i}(\omega) .
$$

Several methods exist to compute the coefficients $\tilde{c}_{i}$; here we focus on the Galerkin and collocation approaches, analyzed in Sections 3.1 and 3.2, respectively. Both methods are applied to the model problems in Example 1 
by increasing the polynomial degree $P \in \mathbb{N}$, in order to inspect the $L^{\infty}$ convergence rates of polynomial approximation (12) and compare them with the asymptotic theoretical results. ${ }^{2}$

\subsection{Galerkin approach}

Given a finite polynomial basis $\left\{p_{i}\right\}_{i=0}^{P}$, the Galerkin approach finds an approximation (12) of spectral abscissa function (4) such that the residual is orthogonal w.r.t. the polynomial basis, in formula

$$
\left\langle\alpha-\alpha_{P}, p_{i}\right\rangle_{\rho}=0, \quad i=0, \ldots, P .
$$

This leads to $\tilde{c}_{i}=c_{i}$, where the coefficients $c_{i}$ are defined in (6). Hence, the Galerkin approach is nothing else than a truncation up to order $P$ of polynomial series (5). Moreover, it provides the optimal approximation in the $\rho$-norm, i.e.

$$
\left\|\alpha-\alpha_{P}\right\|_{\rho}=\sqrt{\int_{\mathbb{S}}\left(\alpha(\omega)-\alpha_{P}(\omega)\right)^{2} \rho(\omega) \mathrm{d} \omega}
$$

is minimized.

This approximation of $\alpha(\omega)$ corresponds to the stochastic Galerkin approximation of $\alpha(\boldsymbol{\omega})$ in the PC theory.

The analyses of the convergence consider, first of all, the truncation error up to order $P$, and then, the numerical error due to the computation of the coefficients $c_{i}$.

\subsubsection{Approximation error}

Assuming that the coefficients $c_{i}$ are correctly evaluated, the following theorem, derived by [41], determines the error bounds of polynomial approximation (12), obtained by the Galerkin approach with Legendre polynomials $\left\{p_{i}\right\}_{i=0}^{P}$ (with $\rho(\omega)=1 / 2$ for $\omega \in[-1,1]$ ).

Theorem 2 Let $\alpha, \alpha^{\prime}, \ldots \alpha^{(k-1)}$ be absolutely continuous on $\mathbb{S}=[-1,1]$, for $k>1$ and

$$
\int_{-1}^{1} \frac{\left|\alpha^{(k+1)}(\omega)\right|}{\sqrt{1-\omega^{2}}} \mathrm{~d} \omega=V_{k}<\infty
$$

Then, for each $P>k+1$ and $\omega \in \mathbb{S}$, the following relation holds

$$
\left\|\alpha-\alpha_{P}\right\|_{\infty} \leq \frac{V_{k} \sqrt{\pi / 2}}{(k-1)(P-k)^{k-0.5}} .
$$

2 The reader interested in a fully automated procedure for polynomial approximation, which adaptively select the degree $P$ by monitoring the decrease of the magnitude of the coefficients $\tilde{c}_{i}$, is referred to software Chebfun, used for example in our tutorial [9] and in [36]. 
Condition (15) corresponds to the fact that the $k$ th derivative, $\alpha^{(k)}$, presents bounded variation w.r.t. the Chebyshev weight function.

The level of smoothness, i.e. the maximum $k$ satisfying the assumption of Theorem 2, heavily determines the convergence behavior of polynomial approximation (12). Only the SAE case verifies the assumptions of Theorem 2, and it satisfies them for all $k \in \mathbb{N}$, ensuring a convergence rate faster than $\mathcal{O}\left(P^{-k}\right)$, for all $k \in \mathbb{N}$. In fact, the convergence rate for SAE is at least geometric, based on analytic extension of real functions on the complex plane [41]; such extension always exists for a real analytic function.

Considering Example 1, the truncation error obtained by the Galerkin approach for the spectral abscissa of parametric eigenvalue problem (SAE) presents a spectral convergence, $\mathcal{O}\left(P^{-P}\right)$, as shown in Figure 2. The (MSSAEs) and (MNSSAEs) converge with an order of $\mathcal{O}\left(P^{-1}\right)$, and $\mathcal{O}\left(P^{-0.5}\right)$, respectively. The coefficients of the polynomial approximation are analytically evaluated in Appendix A.

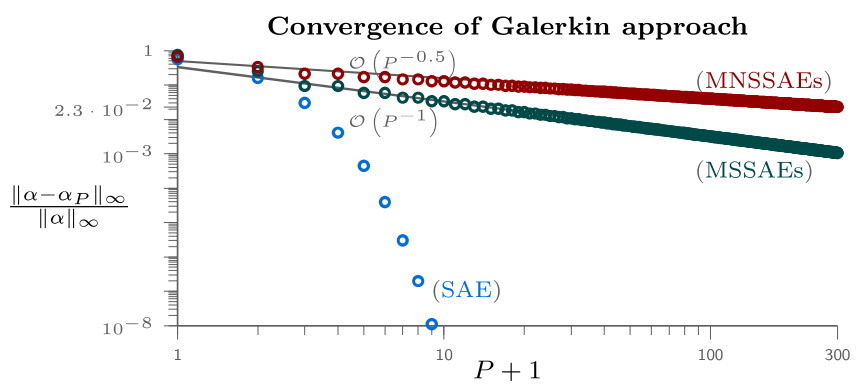

Fig. 2: Relative error of polynomial approximation (12) obtained by the Galerkin approach up to order $P$ of the spectral abscissa associated to the parametric eigenvalue problems of Example 1.

\subsubsection{Numerical error}

It is not always possible to analytically compute the coefficients $c_{i}=\tilde{c}_{i}$ with (6), therefore an integration method based on $M+1$ nodes can approximate the integrals $\left\langle\alpha, p_{i}\right\rangle_{\rho}$ and consequently the coefficients, denoted by $\tilde{c}_{i}^{M}$. This type of approach is known as Non Intrusive Spectral Projection in the PC framework.

In this section, we approximate the coefficients of the Galerkin polynomial approximation $\alpha_{P}^{M}$ with the following integration methods based on $M+1$ points:

Classical integration methods based on an equally spaced discretization of $\mathbb{S}$ containing the extremes of $\mathbb{S}$ : -1 and 1 . In particular, we focus on extended 
trapezoidal rule and extended Simpson's rule. For the latter method, $M$ is required to be an even number.

Interpolatory quadrature rules which approximate an integral by integrating the interpolant of its integrand, where the degree of the polynomial interpolant is at most $M$. We consider Clenshaw-Curtis and Gauss quadrature rules based on Chebyshev and Legendre points, respectively. (Further information on the interpolatory properties of Chebyshev points are given in the upcoming Section 3.2.)

For smooth functions, including the SAE case, the extended trapezoidal and Simpson's rules provide an order of convergence $\mathcal{O}\left(M^{-2}\right)$ and $\mathcal{O}\left(M^{-4}\right)$, respectively.

Figure 3 shows the numerical errors of classical integration methods to evaluate the first coefficient $c_{0}$ of polynomial expansion (5) for Example 1. The convergences for (SAE) follow the theoretical error bounds. For (MSSAEs) and (MNSSAEs), the convergence rates for both classical integration methods are $\mathcal{O}\left(M^{-2}\right)$ and $\mathcal{O}\left(M^{-1.5}\right)$, respectively.

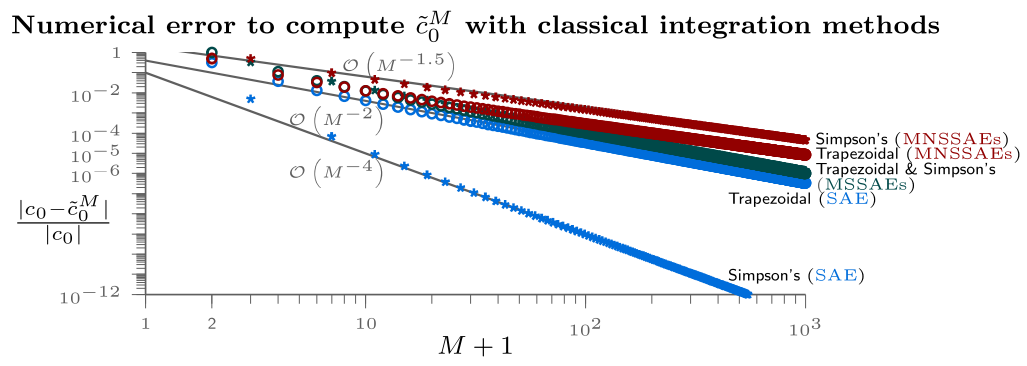

Fig. 3: Error to compute an approximation of the first coefficient $\tilde{c}_{0}^{M}$ of (12) with classical integration methods (extended Trapezoidal and Simpson's rules) based on $M+1$ equally spaced points for problems of Example 1 .

Whenever the integrand is smooth enough to be well-approximated by a polynomial, the interpolatory quadrature rules perform better than the classical integration rule; as stated in the following theorem, combining the results of $[36,44,43]$.

Theorem 3 For an integer $k \geq 1$, let $\alpha, \alpha^{\prime}, \ldots \alpha^{(k-1)}$ be absolutely continuous on $\mathbb{S}=[-1,1]$ and let $\left(\alpha(\omega) p_{i}(\omega)\right)^{(k)}$ present bounded total variation $V_{i}$ with $i \in \mathbb{N}$. Then Clenshaw-Curtis quadrature rule, for the approximation of $c_{i}$ with $M+1$ points, i.e. $\tilde{c}_{i}^{M}$, satisfies

$$
\left|c_{i}-\tilde{c}_{i}^{M}\right| \leq \frac{32}{15} \frac{V_{i}}{\pi k(M-k)^{k}}, \text { for } M>k
$$


and $(M+1)$-Gauss quadralure satisfies

$$
\left|c_{i}-\tilde{c}_{i}^{M}\right| \leq \frac{32}{15} \frac{V_{i}}{\pi k(2 M+1-k)^{k}}, \text { for } M>2 k+1 .
$$

Moreover, for $k=1$, Clenshaw-Curtis quadrature rule presents a convergence rate of $\mathcal{O}\left(M^{-2}\right)$, while Gauss quadrature error is at most of size $\mathcal{O}\left(M^{-2} \ln (M)\right)$

The theorem can be applied to SAE, providing a convergence rate faster than $\mathcal{O}\left(M^{-k}\right)$ for all $k \in \mathbb{N}$, for both interpolatory quadrature rules. Moreover the theorem ensures that in the MSSAEs Clenshaw-Curtis quadrature has a rate of convergence of $\mathcal{O}\left(M^{-2}\right)$, while the Gauss quadrature errors converge, at least, as $\mathcal{O}\left(M^{-2} \ln (M)\right)$.

Remark 1 Theoretical error bounds can be computed also for the particular spectral abscissa (MNSSAEs). Indeed, following the methodology in [44], the Chebyshev coefficients of the spectral abscissa (MNSSAEs) decay as $\mathcal{O}\left(i^{-\frac{3}{2}}\right)$, namely

$$
\frac{2}{\pi} \int_{0}^{\pi / 2} \sqrt{\cos (\vartheta)} \cos (i \vartheta) \mathrm{d} \vartheta=(-1)^{\left\lfloor\frac{i+3}{2}\right\rfloor} \frac{i^{-\frac{3}{2}}}{\sqrt{2 \pi}}+o\left(i^{-\frac{3}{2}}\right), \text { for } i \rightarrow \infty .
$$

The results of $[44,43]$ provide, hence, that Clenshaw-Curtis and Gauss errors for $\tilde{c}_{i}^{M}$ decay as $\mathcal{O}\left(M^{-\frac{3}{2}}\right)$, and $\mathcal{O}\left(M^{-1}\right)$, respectively.

Figure 4 illustrates the numerical error induced by the approximation with interpolatory quadrature rules of the first coefficient $c_{0}$ of Example 1 . (SAE) converges with an order of $\mathcal{O}\left(M^{-M}\right)$, improving the convergence rate of the classical integration methods. For non-smooth cases, (MSSAEs) and (MNSSAEs), the convergence rates of interpolatory quadrature rules are similar to the ones obtained by classical integration methods (Figure 3).

From this numerical experiment, Clenshaw-Curtis and Gauss quadrature rules present similar convergence rates. Hence, the bound of Theorem 3 for the MSSAEs and for the spectral abscissa (MNSSAEs) are optimal for ClenshawCurtis quadrature rule, and conservative for Gauss quadrature rule (as observed in [44] and [43]).

Remark 2 When the coefficients $\tilde{c}_{i}^{M}$ of $\alpha_{P}^{M}$ are approximated by interpolatory quadrature rules, it is advised to set $M \geq P$ as explained with further details in the following Section 4.1.3.

Remark 3 In the PC framework, and in particular for Theorem 1, the first coefficient $\tilde{c}_{0}^{M}$ approximate the mean of the spectral abscissae in Example 1, where $\omega$ is a realization of random variable uniformly distributed in $[-1,1]$. The corresponding convergence rates for the mean (Figures 3 and 4) provide only insights on the numerical error of the integration method, and are not meaningful for the truncation error of the polynomial approximation. 


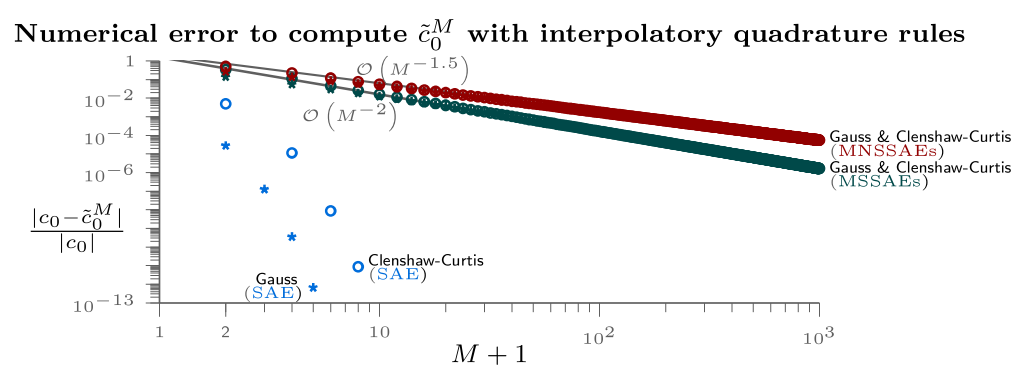

Fig. 4: Numerical error to compute the first coefficient $\tilde{c}_{0}^{M}$ of (12) through Clenshaw-Curtis and Gauss quadrature rules with $M+1$ points for problems of Example 1.

\subsection{Collocation approach}

The collocation approach determines the coefficients of (12) by interpolation on $P+1$ points, $\left\{\omega_{i}\right\}_{i=0}^{P}$ :

$$
\alpha_{P}\left(\omega_{i}\right)=\alpha\left(\omega_{i}\right), \text { for all } i \in\{0, \ldots, P\} .
$$

The coefficients $\tilde{c}_{i}$ of the polynomial approximation $\alpha_{P}$ can be computed solving a linear system of $P+1$ equations, and this can be often done with a negligible numerical error.

Remark 4 The collocation method is mainly affected by approximation errors, while the Galerkin approach might present also a numerical error, due to numerical computation of the coefficients, as illustrated in the previous Section. On the other hand, whereas the Galerkin approach provide a dynamically compute the coefficients, i.e. each coefficient is evaluated independently from the others, the collocation approach simultaneously evaluates all $P+1$ coefficients, solving a linear system of equation.

A widely used choice of interpolating nodes for the interval $[-1,1]$ are Chebyshev nodes. The polynomial interpolant satisfies error bounds, similar to Theorem 2, and provides a near-best approximation in $L^{\infty}$ sense, as stated in the following theorem, whose proof can be found e.g. in [36].

Theorem 4 If $\alpha, \alpha^{\prime}, \ldots \alpha^{(k-1)}$ are absolutely continuous on $\mathbb{S}=[-1,1]$ and $\alpha^{(k)}$ presents bounded total variation $V$ for some $k \geq 1$, then the Chebyshev interpolant $\alpha_{P}$ of degree $P>k$, satisfies

$$
\left\|\alpha-\alpha_{P}\right\|_{\infty} \leq \frac{4 V}{\pi k(P-k)^{k}} .
$$

Moreover, if $\alpha_{P}^{\star}$ is the best polynomial approximation of order less than or equal to $P$, then

$$
\left\|\alpha-\alpha_{P}\right\|_{\infty} \leq\left(2+\frac{2}{\pi} \log (P+1)\right)\left\|\alpha-\alpha_{P}^{\star}\right\|_{\infty} .
$$


Theorem 4 ensures the collocation approach on Chebyshev nodes converge, at least, as $\mathcal{O}\left(P^{-1}\right)$ and faster than $\mathcal{O}\left(P^{-k}\right)$ for all $k \in \mathbb{N}$, for the MSSAEs and SAE cases, respectively.

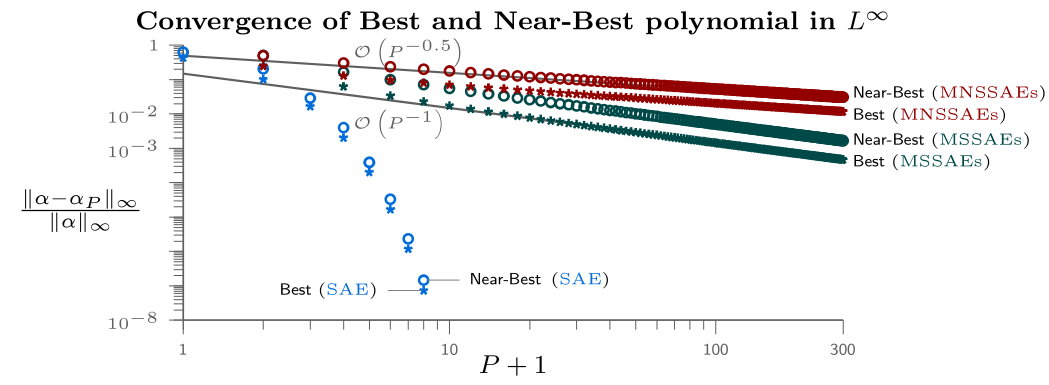

Fig. 5: Relative error of polynomial approximation (12) obtained by interpolation on $P+1$ Chebyshev points (near-best approximation) for the spectral abscissa associated to the parametric eigenvalue problems of Example 1. For completeness the best polynomial approximation in $L^{\infty}$ sense is shown.

The computation of the polynomial interpolant on Chebyshev points can be achieved trough Fast Fourier Transform based algorithm, mapping interpolating values onto coefficients of a polynomial approximation in the Chebyshev polynomial basis. Chebyshev interpolants for the model problems in Example 1 , as well as the best $L^{\infty}$ polynomial approximation are evaluated by Chebfun. Figure 5, other than indicating the interpolating error (near-best polynomial approximation), shows the convergence rates of the best $L^{\infty}$ polynomial approximation. The convergence rates are analogous to the ones obtained by the Galerkin approach (Section 3.1, Figure 2) and agree with Theorem 4 for SAE and MNSSAEs.

Remark 5 In order to apply Theorem 1 to Example 1, considering $\omega$ in is a realization of a uniform distribution in $[-1,1], \boldsymbol{\omega}$, a polynomial transformation is needed. This transformation converts the Chebyshev expansion coefficients into the Legendre coefficients and does not affect the results shown in Figure 5, since the numerical error is negligible [35].

\section{Experiments on the polynomial approximation for $D>1$}

In this section, first of all, we generalize polynomial approximation (12) for $D>1$; in this way the coefficients $\tilde{c}_{i}$ can be evaluated by the Galerkin and collocation approaches, through the corresponding formula (13) and (20). Then, we consider examples of $D=2$ parametric eigenvalue problems, where spectral abscissa functions present the different behaviors characterized in Sec- 
tion 2. The Galerkin and collocation approaches are, hence, applied to the latter benchmark in Sections 4.1 and 4.2, respectively.

We focus on polynomial approximation using a degree graded polynomial basis $\left\{p_{i}(\omega)\right\}_{i=0}^{\infty}$, constructed by products of univariate degree graded polynomial bases $\left\{p_{i, d}\left(\omega_{d}\right)\right\}_{d=1, \ldots, D}^{i=0, \ldots, \infty}$. The degree grading of the polynomial basis $\left\{p_{i}\right\}_{i}$ follows a norm $\|\cdot\|$ on $\mathbb{N}^{D}$, which is associated to a $D$-tupling function $\varphi_{D}$. Therefore, the basis is obtained by formula (9), and the generalization of polynomial approximation (12) for the multivariate case is given by

$$
\alpha_{P}(\omega)=\sum_{\left\|\varphi_{D}^{-1}(i)\right\| \leq P_{d},} \tilde{c}_{i \in \mathbb{N}} p_{i}(\omega)=\sum_{i=0}^{P} \tilde{c}_{i} p_{i}(\omega),
$$

where $P+1$ is equal to the number of polynomials in $\left\{p_{i}\right\}_{i}$ such that the multivariate degree is less than or equal to $P_{d} \geq 0$.

Remark 6 The bijective $D$-tupling functions $\varphi_{D}: \mathbb{N} \rightarrow \mathbb{N}^{D}$ provide an ordering w.r.t each stochastic dimension of the problem, allowing the extension from univariate to multivariate case. Indeed, we are interested in the $D$-tupling functions such that for $i \leq j \varphi_{D}^{-1}(i)=\left(i_{1}, \ldots, i_{D}\right)$ and $\varphi_{D}^{-1}(j)=\left(j_{1}, \ldots, j_{D}\right)$ satisfy $i_{h} \leq j_{h}$ for all $h=1 \ldots, D$. Further details are described in [34].

Two popular choices of norms are the 1-norm, $\left\|\varphi_{D}^{-1}(i)\right\|_{1}=\sum_{d=1}^{D} i_{d}$, and the $\infty$-norm, $\left\|\varphi_{D}^{-1}(i)\right\|_{\infty}=\max _{d=1, \ldots, D} i_{d}$, which are associated to the total and maximal degrees, respectively. In these cases, the number of coefficients $\tilde{c}_{i}$ in $(23)$ satisfies $P+1=\left(\begin{array}{c}P_{d}+D \\ D\end{array}\right)$ and $P+1=\left(P_{d}+1\right)^{D}$ for the total and maximal degree, respectively. Additional information on multivariate degree can be found in [37].

If the bases $\left\{p_{i, d}\left(\omega_{d}\right)\right\}_{d, i}$ are orthogonal w.r.t. $\rho_{d}\left(\omega_{d}\right)$ with $\omega_{d} \in \mathbb{S}_{d} \subset$ $\mathbb{R}$, then the $D$-dimensional polynomial basis $\left\{p_{i}(\omega)\right\}_{i=0}^{\infty}$ is orthogonal to the normalized weight function

$$
\rho(\omega)=\prod_{d=1}^{D} \rho_{d}\left(\omega_{d}\right), \quad \omega \in \mathbb{S}=\underset{d=1}{\times} \mathbb{S}_{d} \subset \mathbb{R}^{D} .
$$

In the PC theory, probability density function (24) corresponds to the assumption that the random vector $\boldsymbol{\omega}$ is constituted by $D$ independent random variable $\boldsymbol{\omega}_{d}, d=1, \ldots, D$. In this framework, the multivariate polynomial degree determines the truncation scheme, and in particular, the total degree corresponds to the standard truncation scheme.

The upcoming Sections 4.1 and 4.2 applies the Galerkin and collocation approaches to the following benchmark examples with $D=2$ uncertain parameters, derived in stabilization of linear time delay system.

Example 2 We consider the spectral abscissa functions associated to the oscillator with feedback delay system

$$
\ddot{x}(t)=-\omega_{1}^{2} x(t)-2 \omega_{1} \omega_{2} \dot{x}(t)+K_{1} x(t-1)+K_{2} \dot{x}(t-1),
$$


such that $x(t)$ is the normalized position at time $t \in[-1, \infty), \omega_{1} \in \mathbb{S}_{1}=$ $[0.9,1.1]$ and $\omega_{2} \in \mathbb{S}_{2}=[0.1,0.2]$ are angular frequency and damping ratio, respectively, while $\left(K_{1}, K_{2}\right)$ describes the control force which acts with a delay of $\tau=1$. The control variables, reported in Table 1 , provide the different spectral abscissa function behaviors (Section 2) for parametric eigenvalue problems with $D=2$. In particular, the MNSSAEs controller is designed by minimizing the spectral abscissa, while the MSSAEs by a more robust stabilization method, namely minimizing the mean of the spectral abscissa [8].

Table 1: Numerical values of the control parameters $K_{1}, K_{2}$ for system (25) corresponding to different behaviors of the spectral abscissa for $\left(\omega_{1}, \omega_{2}\right) \in$ $\mathbb{S}_{1} \times \mathbb{S}_{2}$. (The table is equal to Table 2 in $[8]$ )

\begin{tabular}{cccc}
\hline & SAE & MSSAEs & MNSSAEs \\
\hline$K_{1}$ & 0.2 & 0.5105 & 0.6179 \\
$K_{2}$ & 0.2 & -0.0918 & -0.0072 \\
\hline
\end{tabular}

For this problem, the spectral abscissa functions are not known analytically, even though it is possible to compute their values given $\omega \in \mathbb{S}=\mathbb{S}_{1} \times \mathbb{S}_{2}$. In particular, the convergence rates in $L^{\infty}$ of Figures 7, 9, and 10 are computed w.r.t. $10^{6}$ equidistant points in $\mathbb{S}$.

Without loss of generality, the linear transformation $[-1,1]^{2} \rightarrow \mathbb{S}$ is embedded in system (25), and in the associated parametric eigenvalue problems. Therefore, polynomial bases, interpolation and cubature rules are not shifted and rescaled to $\mathbb{S}$.

The degree grading of the polynomial basis, considered in the following sections, are specified by:

Total Degree is determined by the 1-norm, such that $\left\|\left(i_{1}, i_{2}\right)\right\|_{1}=i_{1}+i_{2}$.

The total degree is associated with a pairing function (i.e. a $D=2$-tupling function) assigning consecutive numbers to points along diagonals of $\mathbb{N} \times \mathbb{N}$, as the Cantor pairing function $\pi_{1}\left(i_{1}, i_{2}\right)$ represented in the left pane of Figure 6 and defined by

$$
\pi_{1}\left(i_{1}, i_{2}\right)=\frac{i_{1}^{2}+3 i_{1}+2 i_{1} i_{2}+i_{2}+i_{2}^{2}}{2} .
$$

The polynomial approximation, obtained from the basis corresponding to the pairing function $\pi_{1}$, i.e. $\left\{p_{i}^{[t]}\right\}_{i}$, such that the polynomials $p_{i}^{[t]}$ have total degree less than or equal to $P_{d}$, is

$$
\alpha_{P}^{[t]}(\omega)=\sum_{\left\|\pi_{1}^{-1}(i)\right\|_{1} \leq P_{d}, i \in \mathbb{N}} \tilde{c}_{i}^{[t]} p_{i}^{[t]}(\omega)=\sum_{i=0}^{P} \tilde{c}_{i}^{[t]} p_{i}^{[t]}(\omega)
$$

where $P+1=\left(\begin{array}{c}P_{d}+2 \\ 2\end{array}\right)=\frac{\left(P_{d}+1\right)^{2}}{2}+\frac{\left(P_{d}+1\right)}{2}$. From (26), we have $P=\pi_{1}\left(P_{d}, 0\right)$. 
Maximal Degree is determined by the $\infty$-norm, such that $\left\|\left(i_{1}, i_{2}\right)\right\|_{\infty}=\max \left\{i_{1}, i_{2}\right\}$. A pairing function, associated to the maximal degree, assigns consecutive numbers to points along the edges of squares of $\mathbb{N} \times \mathbb{N}$, e.g. the RosenbergStrong pairing function $\pi_{\infty}\left(i_{1}, i_{2}\right)$ illustrated in the right pane of Figure 6 and specified by

$$
\pi_{\infty}\left(i_{1}, i_{2}\right)=\left(\max \left\{i_{1}, i_{2}\right\}\right)^{2}+\max \left\{i_{1}, i_{2}\right\}+i_{1}-i_{2} .
$$

The polynomial approximation obtained from the corresponding basis $\left\{p_{i}^{[m]}\right\}_{i}$, such that the polynomials $p_{i}^{[m]}$ have maximal degree less than or equal to $P_{d}$ is

$$
\alpha_{P}^{[m]}(\omega)=\sum_{\left\|\pi_{\infty}^{-1}(i)\right\|_{\infty} \leq P_{d}, i \in \mathbb{N}} \tilde{c}_{i}^{[m]} p_{i}^{[m]}(\omega)=\sum_{i=0}^{P} \tilde{c}_{i}^{[m]} p_{i}^{[m]}(\omega),
$$

where $P+1=\left(P_{d}+1\right)^{2}$. From (28), we have $P=\pi_{\infty}\left(P_{d}, 0\right)$.
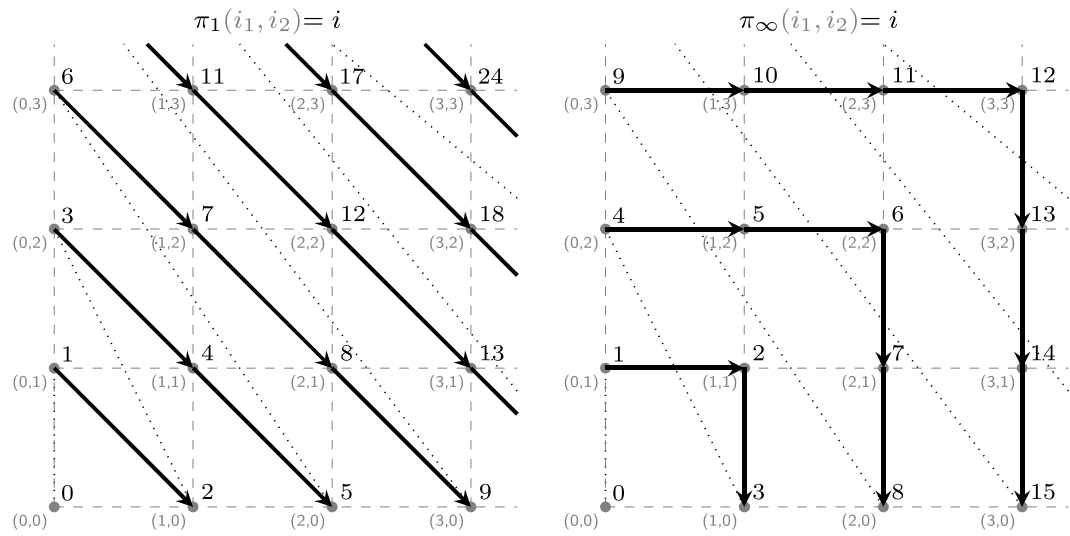

Fig. 6: (Left pane) Cantor pairing function (26). (Right pane) RosenbergStrong pairing function (28).

\subsection{Galerkin approach}

In this section, the spectral abscissa functions associated to (25) with control parameters of Table 1, are approximated by the Galerkin approach on total and maximal Legendre tensor basis, $\left\{p_{i}^{[t]}\right\}_{i}$ and $\left\{p_{i}^{[m]}\right\}_{i}$ with $\rho(\omega)=1 / 4$ for $\omega \in[-1,1]^{2}$. Along the same line of Section 3.1 , we firstly consider the approximation error of the Galerkin approach, evaluating the coefficients with a 
numerical error w.r.t. the approximation error; then, we approximate the coefficients, $\tilde{c}_{i}^{M}$, via integration methods and analyze the numerical error. At the end of this section, some advice to set the different parameters of this approach are given, so that the numerical error does not dominate the approximation error.

\subsubsection{Approximation error}

Figure 7 shows the convergence rates of Galerkin approach approximation error. In this simulation, the coefficients have a negligible numerical error compared to the approximation error, thanks to the advice of the upcoming Section 4.1.3. For both total and maximal degrees, the converge rates are analogous, and they are approximately of order $\mathcal{O}\left(P_{d}^{-P_{d}}\right), \mathcal{O}\left(P_{d}^{-1}\right)$ and $\mathcal{O}\left(P_{d}^{-0.3}\right)$, for the SAE, MSSAEs, and MNSSAEs cases in Example 2, respectively.
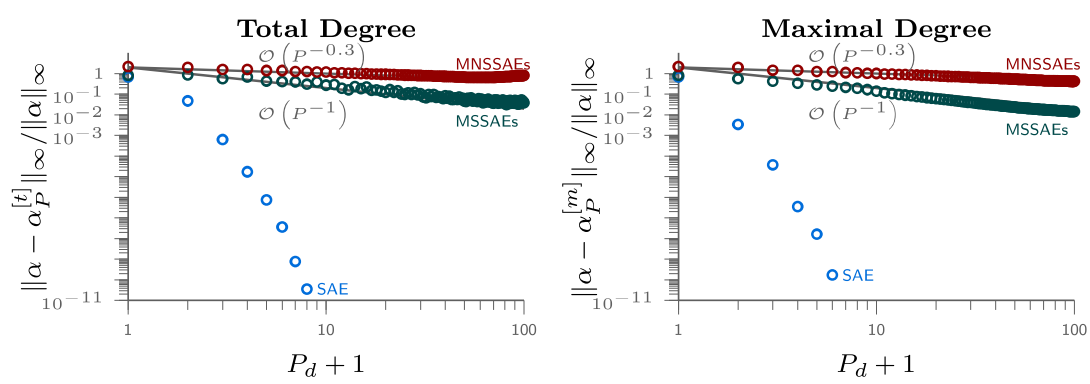

Fig. 7: Approximation errors of the Galerkin approach to compute the polynomial approximation w.r.t. total degree $\alpha_{P}^{[t]}$ and maximal degree $\alpha_{P}^{[m]}$, for the benchmark in Example 2.

\subsubsection{Numerical error}

Bi-dimensional generalizations of the Clenshaw-Curtis quadrature rule based on Chebyshev points provide integral methods to evaluate the coefficients in the Galerkin approach (left hand side of (6)). The integration methods, here considered, are based on the following set of points:

Tensor product Chebyshev grid is constructed by tensor product of $\left(m_{c}+1\right)$ unidimensional Chebyshev points. The total number of points is $M+1=$ $\left(m_{c}+1\right)^{2}$.

Padua points are the self-intersections and boundary contacts of the generating curve $T_{m_{p}}(x)-T_{m_{p}+1}(y)=0$ for $(x, y) \in[-1,1]^{2}$, where $T_{m_{p}}$ is the Chebyshev polynomial of degree $m_{p}$ (cf., e.g., $[2,5]$ and references therein). The total number of points is $M+1=\left(\begin{array}{c}m_{p}+2 \\ 2\end{array}\right)$. 
The approximation of an integral, obtained by integrating the interpolant of the integrand, evaluated on the previous sets of points, leads to the following integration methods (cf. e.g. [33])

Tensorial Clenshaw-Curtis cubature rule based on tensor product Chebyshev grid.

Non-tensorial Clenshaw-Curtis cubature rule relying on Padua points.

These methods approximate the coefficients $\tilde{c}_{i}^{[t], M}$ and $\tilde{c}_{i}^{[m], M}$ and, hence, permit to compute the polynomial approximations w.r.t. total and maximal degrees, i.e. $\alpha_{P}^{[t], M}$ and $\alpha_{P}^{[m], M}$, respectively. By the advice furnished in the upcoming Section 4.1.3, the coefficients $\tilde{c}_{i}^{[t], M}$ are computed by non-tensorial Clenshaw-Curtis cubature rules on $M+1$ Padua points, while $\tilde{c}_{i}^{[m], M}$ are evaluated by tensorial Clenshaw-Curtis cubature rules on $M+1$ tensor product Chebyshev grid.

In order to compute the numerical errors, $\tilde{c}_{i}^{[t], M^{\star}}$ and $\tilde{c}_{i}^{[m], M^{\star}}$ are considered as reference values. These coefficients are computed by the corresponding cubature rules based on $M^{\star}>5 \cdot 10^{5}$ points, in particular $m_{p}=999$ and $m_{c}=707$. Figure 8 shows the error to compute the first coefficient $\tilde{c}_{0}^{M}$, which is independent from the multivariate polynomial degree, since $c_{0}^{[t]}=c_{0}^{[m]}$. The cubature rules on tensor product Chebyshev grid and Padua points present similar convergences rates; in particular the MSSAEs and MNSSAEs converge as $\mathcal{O}\left(M^{-2}\right)$ and $\mathcal{O}\left(M^{-1.5}\right)$, respectively; while the SAE present a convergence of order $\mathcal{O}\left(M^{-M}\right)$.

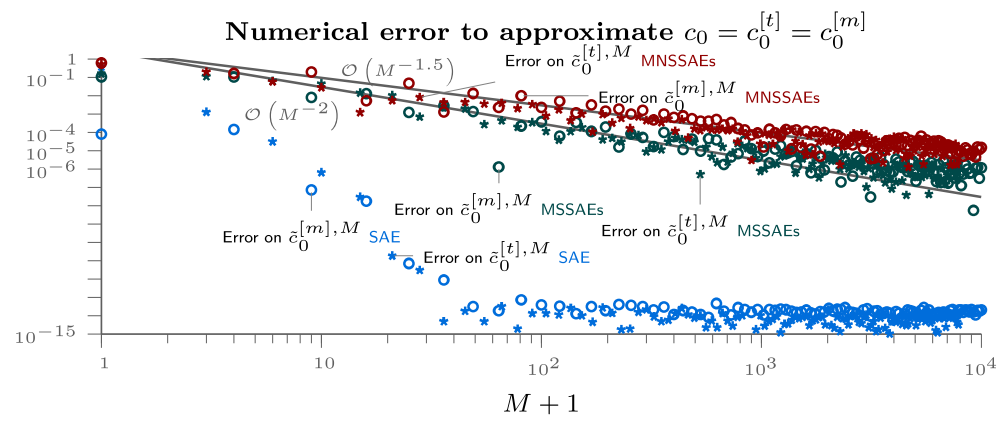

Fig. 8: Numerical error to compute the first coefficient $c_{0}$ of (23) for benchmark in Example 2. The approximations $\tilde{c}_{0}^{[t], M}$, and $\tilde{c}_{0}^{[m], M}$ are obtained by non-tensorial and tensorial Clenshaw-Curtis cubature rules, respectively. The reference values are computed by $M^{\star}>5 \cdot 10^{5}$ which corresponds to $m_{p}=999$ and $m_{c}=707$.

In terms of a PC expansion (see Theorem 1), Figure 8 corresponds to the error of the mean of the spectral abscissa $\alpha(\boldsymbol{\omega})$ associated to $(25)$, where $\omega$ is 
a realization of $\boldsymbol{\omega}$, random vector uniformly distributed in $\mathbb{S}$. For this example, the tensorial and non-tensorial Clenshaw-Curtis cubature rules provide a faster convergence rate of the mean w.r.t. Quasi Monte Carlo method based on Halton sequences, which converges with an order of $\mathcal{O}\left(M^{-1}\right)$ for all the three cases (SAE, and MSSAEs and MNSSAEs) as shown in the left pane of Figure 4 in [8].

\subsubsection{Decoupling numerical error w.r.t. approximation error}

This section furnishes some advice on how to set the number of points in the integration methods, to accurately compute the coefficients of the Galerkin approach. These integration methods should consider a small number of points, $M$, which are still sufficiently large to avoid the corruption of the approximation error by the numerical error, due to the approximation of the integrals.

Since the coefficients $c_{i}$ in (12) and (23) are determined by integrating $\alpha(\omega) p_{i}(\omega) \rho(\omega)$ over the domain $\omega \in \mathbb{S}$, we request that the polynomial approximation behind the integration rule is exact for all polynomials $\left\{p_{i}\right\}_{i=0}^{P}$.

This advice leads in the unidimensional case (Section 3.1.2) that the nodes number in the interpolatory quadrature rules (i.e. Gauss and Clenshaw-Curtis quadrature rules), $M+1$, is greater than the degree of the polynomial approximation $\alpha_{P}^{M}$, i.e. $P$.

For the bi-dimensional case, the polynomial approximation behind the non-tensorial Clenshaw-Curtis cubature rule, i.e. the interpolation on Padua points, is exact for polynomial with total degree less than or equal to $m_{p}$. The interpolant on tensor product Chebyshev grid, underling the tensorial Clenshaw-Curtis cubature rule, is exact for all the polynomials with maximal degree less than or equal to $m_{c}$. From these properties, the interpolatory cubature rule for $D>1$ are associated to the polynomial multivariate degree. In particular, non-tensorial and tensorial Clenshaw-Curtis cubature rules are interpolatory cubature rules associated to the total and maximal degrees for $D=2$, respectively.

Following the advice, $\alpha_{P}^{[t], M}$ is accurately approximated by non-tensorial Clenshaw-Curtis cubature rule with $m_{p} \geq P_{d}$ or by tensorial Clenshaw-Curtis cubature rule with $m_{c} \geq P_{d}$. On the other hand, $\alpha_{P}^{[m], M}$ can be evaluated by tensorial Clenshaw-Curtis with $m_{c} \geq P_{d}$ or by non-tensorial ClenshawCurtis cubature rule with $m_{p} \geq 2 P_{d}$. Therefore, the nodes number in the integration rule is minimized by considering non-tensorial Clenshaw-Curtis cubature rule for approximating the coefficients of $\alpha_{P}^{[t], M}$ with $m_{p} \geq P_{d}$, and tensorial Clenshaw-Curtis cubature rule for approximating the coefficients of $\alpha_{P}^{[m], M}$ with $m_{c} \geq P_{d}$.

Table 2 summarizes the advice for the bi-dimensional case, highlighting the natural choices of computing the polynomial approximations $\alpha_{P}^{[t], M}$ and $\alpha_{P}^{[m], M}$ through the associated interpolatory cubature rules. These natural choices provide the convergence errors in Figure 7, where the coefficients of 
Table 2: Advice to compute Galerkin polynomial approximations (27) and (29), whose coefficients are evaluated by non-tensorial and tensorial ClenshawCurtis cubature rule, based on Padua points and tensor product Chebyshev grid, respectively. The natural choices are highlighted.

\begin{tabular}{|c|c|c|}
\hline & Total degree $\alpha_{P}^{[t], M}$ & Maximal degree $\alpha_{P}^{[m], M}$ \\
\hline Padua points & $\begin{aligned} & m_{p} \geq P_{d} \\
& M+1 \geq P+1 \\
&\left(m_{p}+2\right. \\
&\left.{ }_{2}\right) \geq\left(\begin{array}{c}P_{d}+2 \\
2\end{array}\right)\end{aligned}$ & 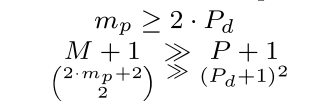 \\
\hline $\begin{array}{l}\text { Tensor product } \\
\text { Chebyshev grid }\end{array}$ & $\begin{aligned} m_{c} & \geq P_{d} \\
M+1 & \gg P+1 \\
\left(m_{c}+1\right)^{2} & \gg\left(\begin{array}{l}\left.P_{d_{2}+2}\right) \\
n_{2}\end{array}\right.\end{aligned}$ & $\begin{aligned} m_{c} & \geq P_{d} \\
M+1 & \geq P+1 \\
\left(m_{c}+1\right)^{2} & \geq\left(P_{d}+1\right)^{2}\end{aligned}$ \\
\hline
\end{tabular}

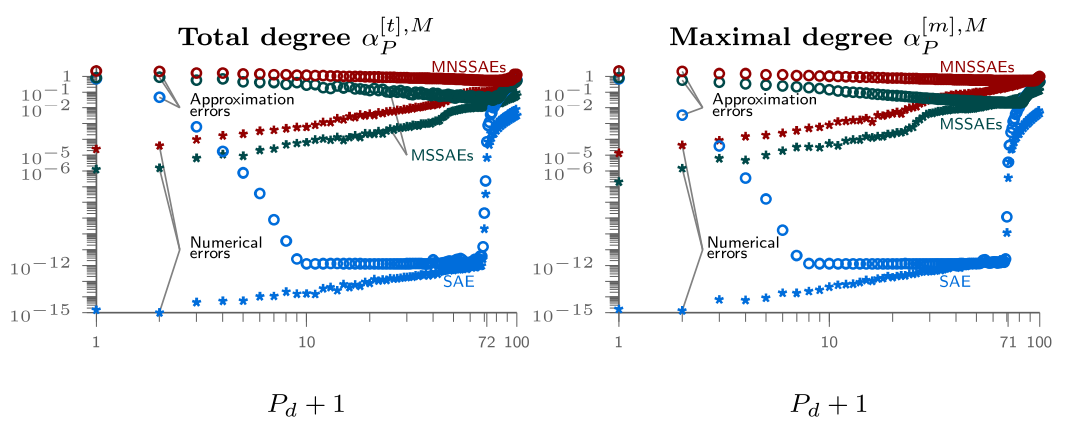

Fig. 9: Numerical and approximation errors to compute the polynomial approximation by the Galerkin approach of the spectral abscissa functions of Example 2, using the two non-natural choices of Table 2 . The coefficients $\tilde{c}_{i}^{[t], M}$ and $\tilde{c}_{i}^{[m], M}$ are computed by tensorial and non-tensorial Clenshaw-Curtis cubature rules, respectively, where $M>P$ is given by $m_{c}=71<100$ and $m_{p}=140<2 \cdot 100$.

$\alpha_{P}^{[t]}, \alpha_{P}^{[m]}$ are evaluated with $m_{p}=110$ non-tensorial and $m_{c}=110$ tensorial Clenshaw-Curtis cubature rule, respectively. On the other hand, Figure 9 shows a counter check of this advice for spectral abscissa polynomial approximations of Example 2. The number of points of the cubature rules $M$ are greater than the number of coefficients, $P$; however, the polynomial approximation $\alpha_{P}^{[t], M}$ is computed though tensorial Clenshaw-Curtis cubature with $m_{c}=71$ while the polynomial approximation $\alpha_{P}^{[m], M}$ is evaluated by nontensorial Clenshaw-Curtis cubature rule with $m_{p}=140$. The numerical errors

$$
\sum_{\left\|\pi_{1}^{-1}(i)\right\|_{1} \leq P_{d}}\left|c_{i}^{[t], M^{\star}}-\tilde{c}_{i}^{[t], M}\right| \quad \text { and } \sum_{\left\|\pi_{\infty}^{-1}(i)\right\|_{\infty} \leq P_{d}}\left|c_{i}^{[m], M^{\star}}-\tilde{c}_{i}^{[m], M}\right|
$$


heavily affect the approximation errors as soon as $P_{d} \gtrsim m_{c}=71$ for $\alpha_{P}^{[t], M}$, and $P_{d} \gtrsim \frac{m_{p}}{2}=70$ for $\alpha_{P}^{[m], M}$.

\subsection{Collocation approach}

Contrary to the Galerkin approach, where the coefficients can be computed independently one of each other with formula (6), in the collocation approach the number of degree of freedom, i.e. the $P+1$ coefficients, should match the number of interpolation points. Hence the interpolant of total degree $P_{d}$, $\alpha_{P}^{[t]}$, is computed on Padua points with $m_{p}=P_{d}$, while the interpolant of maximal degree $P_{d}, \alpha_{P}^{[m]}$, is evaluated on tensor products Chebyshev grid with $m_{c}=P_{d}$. The matching between interpolant polynomial and interpolating points is strengthened by the following theorem, which provides the near-best optimal approximation in $L^{\infty}$ associated to a multivariate polynomial degree.

Theorem 5 Let $\alpha_{P}^{[t]^{\star}}$, and $\alpha_{P}^{[t]}$, be the best polynomial approximation of total degree less than or equal to $P_{d}$ and the polynomial interpolant on $m_{p}=P_{d}$ Padua points, respectively. Then

$$
\left\|\alpha-\alpha_{P}^{[t]}\right\|_{\infty} \leq\left(1+\mathcal{O}\left(\log ^{2}\left(P_{d}\right)\right)\right)\left\|\alpha-\alpha_{P}^{[t]^{\star}}\right\|_{\infty} .
$$

Analogously, if $\alpha_{P}^{[m]^{\star}}$, and $\alpha_{P}^{[m]}$, are the best polynomial interpolant of maximal degree less than or equal to $P_{d}$ and the polynomial interpolant on $m_{c}=P_{d}$ tensor product Chebyshev grid, respectively, then

$$
\left\|\alpha-\alpha_{P}^{[m]}\right\|_{\infty} \leq\left(1+\mathcal{O}\left(\log ^{2}\left(P_{d}\right)\right)\right)\left\|\alpha-\alpha_{P}^{[m]^{\star}}\right\|_{\infty}
$$

The near-best optimality is derived by the growth of the Lebesgue constant [2]. Moreover, the following theorem [5] extends the error bounds of Theorem 4 for total degree polynomial interpolant evaluated in Padua points.

Theorem 6 If $\alpha$ is continuous and differentiable up to the $k$ th derivative in $[-1,1]^{2}$, with $0<k<\infty$, then the interpolant $\alpha_{P}^{[t]}$ on $m_{p}=P_{d}$ Padua points satisfies the following relation

$$
\left\|\alpha-\alpha_{P}^{[t]}\right\|_{\infty} \leq \mathcal{O}\left(\frac{\left.\log ^{2}\left(P_{d}\right)\right)}{P_{d}^{k}}\right) .
$$

Theorem 6 provides an error bounds only for the SAE case, ensuring a convergence faster than $\mathcal{O}\left(P_{d}^{-k}\right)$ for all $k \in \mathbb{N}$.

Figure 10 shows the experiments on the spectral abscissa functions of Example 2, for which we used the software Chebfun relying on Chebyshev expansion. The convergence rates are analogous to the ones of the Galerkin approach (Figure 7) the only difference is that the numerical error is negligible for the collocation approach. 

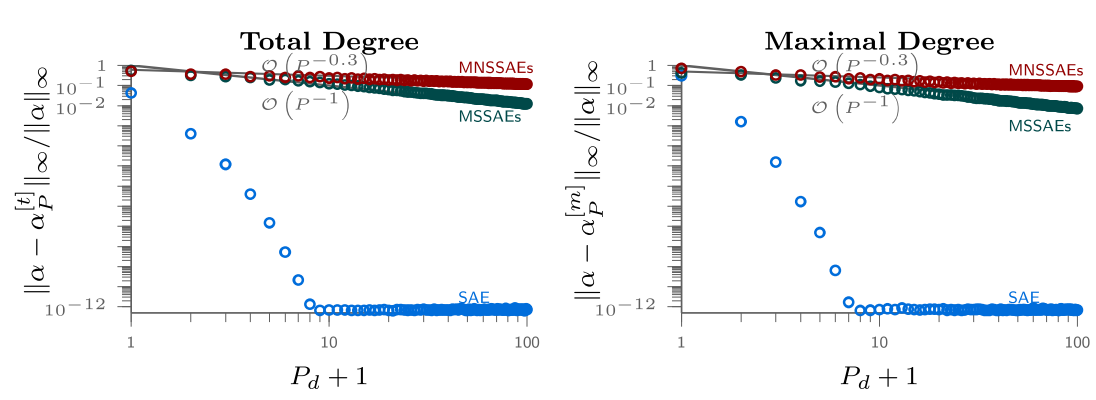

Fig. 10: Convergence rates of interpolant polynomial for collocation approach of the spectral abscissa functions of Example 2, based on Padua points for total degree and tensor product Chebyshev grid for maximal degree.

\section{Perspectives, implications and conclusions}

In this last section, perspective and implications of the main results are discussed. In particular, in the first part, we consider extensions to parametric eigenvalue problem arising from neutral delay differential equations. Secondly, for cases where polynomial approximations are less suitable due to a lack of smoothness of the spectral abscissa, we outline the potential of projection methods for large-scale parametric eigenvalue problem. We end this section with the conclusions.

\subsection{Other classes of delay equation}

The analysis conducted in this text can be extended to general eigenvalue problem presenting a finite number of active eigenvalues in the uncertain domain $\mathbb{S}$. However, this property does not hold in general for the spectrum associated to a time delay system of neutral type. The evolution of these neutral systems not only depends on the solution in the past, as the retarded delay differential equations (2), but also on the derivative of the solution at past time-instants. An important issue in extending the aforementioned analysis to neutral time delay system regards the possible non-continuity of the spectral abscissa, which might not be a continuous function of the delay parameters, as shown in Example 1 in [21].

When there is only one delay or when delays are not affected by uncertainty, the spectral abscissa is continuous. Still, the spectrum associated to a neutral time delay system can present an infinite number of eigenvalues in a given right half plane; therefore the spectrum abscissa might correspond to the supremum of the rightmost eigenvalues' real parts, which is not a maximum. For example, the neutral time delay system $\dot{x}(t)+\dot{x}(t-1)=-2 x(t)-x(t-1)$ has all the eigenvalues in the open left half plane, however the spectral abscissa, defined by the supremum, is zero; this spectrum, analyzed in [39], is illustrated in 
Figure 11. As a consequence, the spectral abscissa may not be described by the behavior of the active eigenvalues, like in Section 2; and branches of infinitely many rightmost eigenvalues might cross each other in the complex plane.

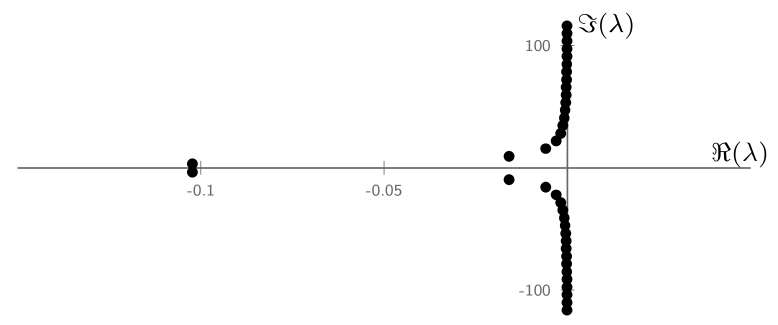

Fig. 11: Spectrum of the neutral delay differential equation $\dot{x}(t)+\dot{x}(t-1)=$ $-2 x(t)-x(t-1)$. In this case, the spectral abscissa corresponds to the supremum of the eigenvalues' real parts, and not to the maximum as in (3).

These phenomena occur also in the spectrum of delay differential algebraic equations (DDAEs), which allow in fact to model both retarded and neutral systems. DDAEs provide a general framework to describe interconnected system and are therefore often considered in system and control [21]. Even though the complex behavior of the eigenvalues of DDAEs exceed in general the purpose of this text, this analysis is applicable to DDAEs of retarded type, since their associated eigenvalue problems present finitely many eigenvalues in any right half-plane [8].

\subsection{Beyond polynomial (chaos) approximations}

This study highlights that maximum eigenvalue functions cannot always be suitably approximated by polynomial (chaos) based method, especially if MSSAEs and MNSSAEs cases are expected or favored, as in system and control applications as described in Section 1 and illustrated by the non-smooth behaviors of Example 2. In this context, also piecewise-polynomial approximation [31] and enriched polynomial approximation [12] are not expected to provide satisfactory solution, since the location of the discontinuities is not known a priori; and for stochastic dimension $D>1$, the geometry of non-differentiable points within $\mathbb{S}$ is not well understood.

For large-scale eigenvalue problems, obtaining an accurate polynomial (chaos) approximations of non-smooth maximum eigenvalue functions becomes infeasible from a computational point of view. In this context, approximations potentially better tailored to the behavior of maximum eigenvalue functions are constituted by projection methods, where a large scale parametric eigenvalue problem is projected into a small one. The advantage is that the approximation is still in the form of the solution of a (small) eigenvalue problem, 
inheriting all properties, and able to preserve the spectral abscissa behaviors of the MSSAEs and MNSSAEs cases. Projection methods are reliably applied in the eigenvalue computations and model reduction [24], as well as eigenvalue optimization [15]; however, their potential for global approximations of maximum eigenvalue functions and computing statical information has not been deeply considered to the best of out knowledge.

We project a generalized eigenvalue problem $A(\omega) v=\lambda B(\omega) v$, where $A(\omega)$, $B(\omega)$ are two non-singular parametric matrices, into the subspace formed by left and right eigenvectors associated to rightmost eigenvalues for given $\omega \in\left\{\omega_{i}\right\}_{i}$. The eigenvalues of the projected eigenproblem provide an Hermite interpolation of the dominant eigenvalues of the original problem at $\omega \in\left\{\omega_{i}\right\}_{i}$, as stated in the following proposition, which extends to generalized eigenvalue problem the results in $[29,15]$.

Proposition 1 () Let $V$ and $W$ be matrices whose column space respectively contains the right and left eigenvectors associated to the rightmost eigenvalues of $A(\omega) v=\lambda B(\omega) v$ for $\omega \in\left\{\omega_{i}\right\}_{i}$. For each $\omega \in\left\{\omega_{i}\right\}_{i}$ if the rightmost eigenvalue $\lambda_{R M}$ is simple and the matrices $A(\omega)$, and $B(\omega)$ are differentiable towards $\omega$, then an eigenvalue of the projected eigenproblem $W^{*} A(\omega) V^{*} \tilde{v}=$ $\lambda W^{*} B(\omega) V^{*} \tilde{v}$ interpolates the original rightmost eigenvalue, and also its derivative w.r.t. the elements of parameter vector $\omega$.

Proof. For notational convenience, set $\omega \in\left\{\omega_{i}\right\}_{i}$ and consider $A(\omega)=A$, $B(\omega)=B$. The left and right eigenvectors associated to the dominant eigenvalues can be rewritten by using the matrices $W$ and $V$, namely $v_{\mathrm{RM}}=V \tilde{v}_{\mathrm{RM}}$ and $w_{\mathrm{RM}}=W \tilde{w}_{\mathrm{RM}}$. Therefore the interpolation of the rightmost eigenvalues is given by:

$$
\lambda_{\mathrm{RM}} W^{*} B V \tilde{v}=W^{*} \lambda_{\mathrm{RM}} B v_{\mathrm{RM}}=W^{*} A v_{\mathrm{RM}}=W^{*} A V \tilde{v}_{\mathrm{RM}} .
$$

Analogous result holds for the derivative of the dominant eigenvalues w.r.t. an element $\hat{\omega}$ of the parameter $\omega$, by using the explicit formula for the derivative of an eigenvalue [8]:

$$
\begin{aligned}
\frac{\partial \lambda_{\mathrm{RM}}}{\partial \hat{\omega}} & =\frac{w_{\mathrm{RM}}^{*} \frac{\partial\left(\lambda_{\mathrm{RM}} B-A\right)}{\partial \hat{\omega}} v_{\mathrm{RM}}}{w_{\mathrm{RM}}^{*} B v_{\mathrm{RM}}}=\frac{\tilde{w}_{\mathrm{RM}}^{*} W^{*}\left(\lambda_{\mathrm{RM}} \frac{\partial B}{\partial \hat{\omega}}-\frac{\partial A}{\partial \hat{\omega}}\right) V \tilde{v}_{\mathrm{RM}}}{\tilde{w}_{\mathrm{RM}}^{*} W^{*} B V \tilde{v}_{\mathrm{RM}}} \\
& =\frac{\tilde{w}_{\mathrm{RM}}^{*} \frac{\partial\left(\lambda_{\mathrm{RM}} W^{*} B V-W^{*} A V\right)}{\partial \hat{\omega}} \tilde{v}_{\mathrm{RM}}}{\tilde{w}_{\mathrm{RM}}^{*} W^{*} B V \tilde{v}_{\mathrm{RM}}} .
\end{aligned}
$$

Remark 7 For symmetric eigenvalue problem, the interpolation properties hold in a stronger sense, i.e. for $\omega \in\left\{\omega_{i}\right\}_{i}$ the rightmost eigenvalue of the original eigenproblem is also the rightmost eigenvalue of the projected eigenproblem. For general real $A, B$ matrices, even though it rarely occurs, the projected eigenproblem may present an active eigenvalue that does not correspond to the eigenvalue of the original problem. 
To illustrate the idea, we consider a generalized parametric eigenvalue problem of dimensions 100 by 100 depending on a scalar parameter $\omega$, obtained from the spectral discretization of the Hayes equation $x^{\prime}(t)=\omega x(t)-0.6 x(t-1)$ with $\omega \in[0,1]$, using the approach of [24]. For this system, the spectral abscissa function presents a MNSSAEs behavior, since for $\omega=0.5$ there is a non-semi-simple active eigenvalue with multiplicity 2 as illustrated in the left pane of Figure 12. We project the generalized eigenproblem into the left and right eigenvectors associated to the rightmost eigenvalues in 3 random points of the interval $[0,1]$; the projected $5 \times 5$ eigenproblem approximate the behaviors of the rightmost eigenvalues of the delay system, as shown in the left pane of Figure $12 .{ }^{3}$ The spectral abscissa $L^{\infty}$ relative error for the projected eigenproblem is $2.2 \cdot 10^{-8}$, six orders of magnitude less than the collocation approximation with 100 points. In addition, the approximation obtained by the projection is computed faster than evaluating an high degree polynomial approximation; as a result the small size of the projected eigenvalue problem provides a fast surrogate model, which can be used to infer statical information.

Original and projected eigenproblems
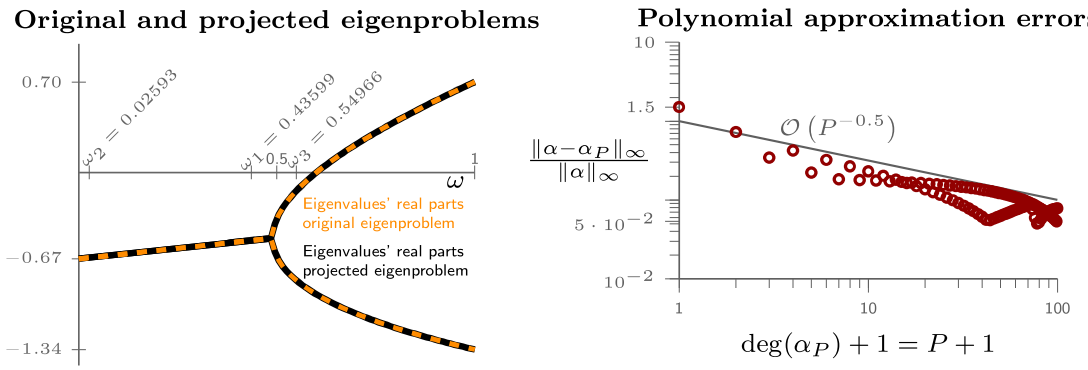

Fig. 12: In the left pane, active eigenvalues' real parts of the projected eigenproblem of dimension $5 \times 5$ mimic the behavior of the active eigenvalues' real part of the original eigenproblem; the relative error of the projected spectral abscissa in $L_{\infty}$ is $2.2 \cdot 10^{-8}$. The projection is based on the left and right eigenvectors of the rightmost eigenvalues in the random points $\omega_{1}, \omega_{2}$, and $\omega_{3}$. In the right pane, the relative errors of the collocation methods on Chebyshev points varying the polynomial degree are shown.

\subsection{Conclusions}

This paper, other than explaining the parallelism between polynomial approximation and PC theory, analyzes the approximation of polynomial series (5)

\footnotetext{
3 If the rightmost eigenvalues are conjugate, the real matrices $V$ and $W$ are constructed considering real as well as imaginary parts of the left and right eigenvectors associated to the active eigenvalue.
} 
of $\alpha(\omega)$ (and its PC expansion (8) of $\alpha(\boldsymbol{\omega})$ ) w.r.t. the behavior of spectral abscissa function (4). The analyses show that the lack of smoothness properties heavily affects both the approximation errors of the Galerkin and collocation approaches, as well as the numerical errors in the approximation of coefficients $c_{i}$ with integration methods.

The convergence rates between the Galerkin and collocation approaches are comparable, whenever numerical errors in the Galerkin approach are negligible. These errors can be neglected by following the advices of Section 4.1.3, which correlate the polynomial degree of $\alpha_{P}^{M}$ with the number of points $M+1$ of the integration methods. In this way the numerical errors, due to integration methods, do not affect the quality of the approximation of the Galerkin approach.

In the test-examples analyzed (Examples 1 and 2), if the spectral abscissa behaves smoothly (SAE), then the polynomial approximation convergences with an order of $\mathcal{O}\left(P_{d}^{-P_{d}}\right)$ for both approaches, where $P_{d}=P$ for $D=1$. However, the non-differentiable and non-Lipschitz continuous behaviors (represented in the benchmark examples by MSSAEs and MNSSAEs) present in the univariate case a order of convergence of $\mathcal{O}\left(P^{-1}\right)$ and $\mathcal{O}\left(P^{-0.5}\right)$, respectively, while in the bivariate case they converge approximately as $\mathcal{O}\left(P_{d}^{-1}\right)$ and $\mathcal{O}\left(P_{d}^{-0.3}\right)$, respectively. These latter cases are not deeply studied in the literature on the spectral abscissa approximation, even though they easily occur when applying stability optimization in control design (cf., e.g. $[10,8]$ and reference therein).

The present work reviews the main theorems on univariate and bivariate polynomial approximation on Chebyshev and Legendre bases for differentiable functions, providing convergence rates for non-differentiable and non-Lipschitz continuous functions, i.e. MSSAEs and MNSSAEs, respectively. Exploiting the parallelism, $L^{\infty}$ theoretical error bounds are applied in the PC framework, providing a novelty in this context. Indeed, the results in PC theory are commonly stated in $L^{2}$ norm, or equivalently to to second order moment, a weaker measure than $L^{\infty}$.

\section{Acknowledgments}

The authors would like to thank A. Bultheel for the careful proofreading and his advice, and L. N. Trefethen for pointing to valuable references.

This work was supported by the project C14/17/072 of the KU Leuven Research Council, by the project G0A5317N of the Research FoundationFlanders (FWO - Vlaanderen), and by the project UCoCoS, funded by the European Unions Horizon 2020 research and innovation program under the Marie Sklodowska-Curie Grant Agreement No 675080. 


\section{References}

1. M. Abramowitz and I. A. Stegun, editors. Handbook of Mathematical Functions. Dover Publications Inc., 1965. ISBN 0486612724.

2. L. Bos, M. Caliari, S. D. Marchi, M. Vianello, and Y. Xu. Bivariate Lagrange interpolation at the Padua points: the generating curve approach. Journal of Approximation Theory, 143(1):15-25, 2006. doi: 10.1016/j.jat.2006.03.008.

3. I. Boussaada, S. Tliba, and S. I. Niculescu. A delayed feedback controller for active vibration control: A rightmost-characteristic root assignment based approach. In 2017 21st International Conference on System Theory, Control and Computing (ICSTCC). IEEE, 2017. doi: 10.1109/icstcc.2017. 8107073.

4. I. Boussaada, S. Tliba, S. I. Niculescu, H. U. Ünal, and T. Vyhlídal. Further remarks on the effect of multiple spectral values on the dynamics of time-delay systems. application to the control of a mechanical system. Linear Algebra and its Applications, 542:589-604, 2018. doi: 10.1016/j.laa.2017.11.022.

5. M. Caliari, S. D. Marchi, and M. Vianello. Bivariate Lagrange interpolation at the Padua points: computational aspects. Journal of Computational and Applied Mathematics, 221(2):284-292, 2008. doi: 10.1016/j.cam.2007.10.027.

6. T. Crestaux, O. L. Maître, and J. M. Martinez. Polynomial chaos expansion for sensitivity analysis. Reliability Engineering \& System Safety, 94 (7):1161-1172, 2009. doi: 10.1016/j.ress.2008.10.008.

7. H. C. Elman and T. Su. Low-rank solution methods for stochastic eigenvalue problems. arXiv, 1803.03717, 2018.

8. L. Fenzi and W. Michiels. Robust stability optimization for linear delay systems in a probabilistic framework. Linear Algebra and its Applications, 526:1-26, 2017. doi: 10.1016/j.laa.2017.03.020.

9. L. Fenzi and W. Michiels. Experiments on polynomial (chaos) approximation of maximum eigenvalue functions: Tutorial. Technical report, TW 688, Department of Computer Science, KU Leuven, 2018.

10. L. Fenzi, D. Pilbauer, W. Michiels, and T. Vyhlídal. A probabilistic approach towards robust stability optimization, with application to vibration control. In F. Stépán and G. Csernák, editors, Proceedings of the 9th European Nonlinear Dynamics Conference, 2017.

11. R. Ghanem and D. Ghosh. Efficient characterization of the random eigenvalue problem in a polynomial chaos decomposition. International Journal for Numerical Methods in Engineering, 72(4):486-504, 2007. doi: $10.1002 /$ nme.2025.

12. D. Ghosh and R. Ghanem. Stochastic convergence acceleration through basis enrichment of polynomial chaos expansions. International Journal for Numerical Methods in Engineering, 73(2):162-184, 2007. doi: 10.1002/ nme.2066. 
13. H. Hakula, V. Kaarnioja, and M. Laaksonen. Approximate methods for stochastic eigenvalue problems. Applied Mathematics and Computation, 267:664-681, 2015. doi: 10.1016/j.amc.2014.12.112.

14. T. Homma and A. Saltelli. Importance measures in global sensitivity analysis of nonlinear models. Reliability Engineering 8 System Safety, 52 (1):1-17, 1996. doi: 10.1016/0951-8320(96)00002-6.

15. F. Kangal, K. Meerbergen, E. Mengi, and W. Michiels. A subspace method for large-scale eigenvalue optimization. SIAM Journal on Matrix Analysis and Applications, 39(1):48-82, 2018. doi: 10.1137/16m1070025.

16. T. Kato. Perturbation Theory for Linear Operators. Springer Berlin Heidelberg, 1995. ISBN 354058661X.

17. J. C. Lan, X. J. Dong, Z. K. Peng, W. M. Zhang, and G. Meng. Uncertain eigenvalue analysis by the sparse grid stochastic collocation method. Acta Mechanica Sinica, 31(4):545-557, 2015. doi: 10.1007/s10409-015-0422-9.

18. K. López, S. Mondié, and R. Garrido. A tuning procedure for the cascade proportional integral retarded controller. IFAC-PapersOnLine, 51(14):6165, 2018. doi: 10.1016/j.ifacol.2018.07.199.

19. O. P. L. Maître and O. M. Knio. Spectral Methods for Uncertainty Quantification. Springer-Verlag GmbH, 2010. ISBN 9048135192.

20. S. Marelli and B. Sudret. UQLab user manual - polynomial chaos expansions. Technical report, Chair of Risk, Safety \& Uncertainty Quantification, ETH Zurich, 2017.

21. W. Michiels. Spectrum-based stability analysis and stabilisation of systems described by delay differential algebraic equations. IET Control Theory $\&$ Applications, 5(16):1829-1842, 2011. doi: 10.1049/iet-cta.2010.0752.

22. W. Michiels and S. I. Niculescu. Stability, Control, and Computation for Time-Delay Systems. Cambridge University Press, 2014. ISBN 1611973627.

23. W. Michiels, K. Engelborghs, P. Vansevenant, and D. Roose. Continuous pole placement for delay equations. Automatica, 38(5):747-761, 2002. doi: 10.1016/s0005-1098(01)00257-6.

24. W. Michiels, E. Jarlebring, and K. Meerbergen. Krylov-based model order reduction of time-delay systems. SIAM Journal on Matrix Analysis and Applications, 32(4):1399-1421, 2011. doi: 10.1137/100797436.

25. W. Michiels, I. Boussaada, and S. I. Niculescu. An explicit formula for the splitting of multiple eigenvalues for nonlinear eigenvalue problems and connections with the linearization for the delay eigenvalue problem. SIAM Journal on Matrix Analysis and Applications, 38(2):599-620, 2017. doi: $10.1137 / 16 \mathrm{~m} 107774 \mathrm{x}$.

26. S. Rahman and V. Yadav. Orthogonal polynomial expansion for solving random eigenvalue problems. International Journal for Uncertainty Quantification, 1(2):163-187, 2011. doi: 10.1615/intjuncertaintyquantification. v1.i2.40.

27. A. Ramirez, S. Mondié, R. Garrido, and R. Sipahi. Design of proportionalintegral-retarded (PIR) controllers for second-order LTI systems. IEEE Transactions on Automatic Control, 61(6):1688-1693, 2016. doi: 10.1109/ 
tac.2015.2478130.

28. A. Ramírez, R. Sipahi, S. Mondié, and R. Garrido. An analytical approach to tuning of delay-based controllers for LTI-SISO systems. SIAM Journal on Control and Optimization, 55(1):397-412, 2017. doi: 10.1137/15m1050999.

29. M. Saadvandi, K. Meerbergen, and W. Desmet. Parametric dominant pole algorithm for parametric model order reduction. Journal of Computational and Applied Mathematics, 259:259-280, 2014. doi: 10.1016/j.cam.2013.09. 012 .

30. E. Sarrouy, O. Dessombz, and J. J. Sinou. Stochastic analysis of the eigenvalue problem for mechanical systems using polynomial chaos expansionapplication to a finite element rotor. Journal of Vibration and Acoustics, 134(5):1-12, 2012. doi: 10.1115/1.4005842.

31. E. Sarrouy, O. Dessombz, and J. J. Sinou. Piecewise polynomial chaos expansion with an application to brake squeal of a linear brake system. Journal of Sound and Vibration, 332(3):577-594, 2013. doi: 10.1016/j.jsv. 2012.09.009.

32. I. M. Sobol. Global sensitivity indices for nonlinear mathematical models and their monte carlo estimates. Mathematics and Computers in Simulation, 55:271-280, 2001. doi: 10.1016/s0378-4754(00)00270-6.

33. A. Sommariva, M. Vianello, and R. Zanovello. Nontensorial Clenshaw-Curtis cubature. Numerical Algorithms, 49:409-427, 2008. doi: 10.1007/s11075-008-9203-x.

34. M. P. Szudzik. The Rosenberg-Strong pairing function. arXiv, 1706.04129, 2017.

35. A. Townsend, M. Webb, and S. Olver. Fast polynomial transforms based on Toeplitz and Hankel matrices. Mathematics of Computation, 87:19131934, 2018. doi: $10.1090 / \mathrm{mcom} / 3277$.

36. L. N. Trefethen. Approximation Theory and Approximation Practice. Cambridge University Press, 2012. ISBN 1611972396.

37. L. N. Trefethen. Multivariate polynomial approximation in the hypercube. Proceedings of the American Mathematical Society, 145(11):48374844, 2017. doi: 10.1090/proc/13623.

38. R. Vermiglio. Polynomial chaos expansions for the stability analysis of uncertain delay differential equations. SIAM/ASA Journal on Uncertainty Quantification, 5(1):278-303, 2017. doi: 10.1137/15m1029618.

39. E. I. Verriest and W. Michiels. Inverse routh table construction and stability of delay equations. Systems \& Control Letters, 55(9):711-718, 2006. doi: $10.1016 /$ j.sysconle.2006.02.002.

40. R. Villafuerte, S. Mondié, and R. Garrido. Tuning of proportional retarded controllers: Theory and experiments. IEEE Transactions on Control Systems Technology, 21(3):983-990, 2013. doi: 10.1109/tcst.2012.2195664.

41. H. Wang and S. Xiang. On the convergence rates of Legendre approximation. Mathematics of Computation, 81(278):861-877, 2011. doi: 10.1090/s0025-5718-2011-02549-4. 
42. M. M. R. Williams. A method for solving stochastic eigenvalue problems II. Applied Mathematics and Computation, 219(9):4729-4744, 2013. doi: 10.1016/j.amc.2012.10.089.

43. S. Xiang. An improved error bound on Gauss quadrature. Applied Mathematics Letters, 58:42-48, 2016. doi: 10.1016/j.aml.2016.01.015.

44. S. Xiang and F. Bornemann. On the convergence rates of Gauss and Clenshaw-Curtis quadrature for functions of limited regularity. SIAM Journal on Numerical Analysis, 50(5):2581-2587, 2012. doi: 10.1137/ 120869845 .

45. D. Xiu. Numerical Methods for Stochastic Computations. Princeton University Press, 2010.

\section{A Galerkin approach for the spectral abscissa functions in Example 1}

The spectral abscissa functions for eigenvalue problems (SAE), (MSSAEs), and (MNSSAEs) are given by

$$
\alpha^{\mathrm{I}}(\omega)=e^{\omega}, \quad \alpha^{\mathrm{II}}(\omega)=\left\{\begin{array}{ll}
0, & \text { if } \omega \in[-1,0), \\
\omega, & \text { if } \omega \in[0,1] ;
\end{array} \quad \alpha^{\mathrm{III}}(\omega)= \begin{cases}0, & \text { if } \omega \in[-1,0), \\
\sqrt{\omega}, & \text { if } \omega \in[0,1]\end{cases}\right.
$$

respectively. In this appendix, we analytically compute the quantities, which appear in the evaluation of the coefficients $c_{i}$ in equation (6).

The $\rho$-norms of the Legendre polynomial are given by $\left\|p_{i}\right\|_{\rho}^{2}=\frac{1}{2 i+1}$ for all $i \in \mathbb{N}$ (see e.g. 22.2 .10 in $[1])$.

To analytically express the coefficients $c_{i}$ in (6), the evaluation of the $\rho$-inner product of spectral abscissae (34) and the $i$ th Legendre polynomials is needed. We start analyzing $\alpha^{\mathrm{I}}(\omega)$ and we furnish the corresponding $\rho$-inner product iteratively, starting from:

$$
\left\langle\alpha^{\mathrm{I}}, p_{0}\right\rangle_{\rho}=\frac{1}{2}\left(e-e^{-1}\right) .
$$

Set $i \in \mathbb{N}$ and $i \geq 1$, by using integration by parts, and the following Legendre polynomial properties

$$
p_{i}( \pm 1)=( \pm 1)^{i}, \quad \text { and } \frac{\mathrm{d} p_{i+1}(\omega)}{\mathrm{d} \omega}=\sum_{k=0}^{\lfloor i / 2\rfloor} \frac{p_{i-2 k}(\omega)}{\left\|p_{i-2 k}\right\|_{\rho}^{2}}, \quad \text { where }\left\lfloor\frac{i}{2}\right\rfloor=\max _{j \in \mathbb{N}}\left\{j \leq \frac{i}{2}\right\},
$$

we get

$$
\left\langle\alpha^{\mathrm{I}}, p_{i+1}\right\rangle_{\rho}=\frac{1}{2}\left(e+\frac{(-1)^{i}}{e}-2 \sum_{k=0}^{\lfloor i / 2\rfloor} \frac{\left\langle\alpha^{\mathrm{I}}, p_{i-2 k}\right\rangle_{\rho}}{\left\|p_{i-2 k}\right\|_{\rho}^{2}}\right)=\frac{1}{2}\left(e+\frac{(-1)^{i}}{e}-2 \sum_{k=0}^{\lfloor i / 2\rfloor} c_{i}^{\mathrm{I}}\right),
$$

where $c_{i}^{\mathrm{I}}$ indicates the $i$ th coefficients of polynomial approximation (5) of $\alpha^{\mathrm{I}}(\omega)$.

For all $i \in \mathbb{N}$, the $\rho$-inner product of the spectral abscissae $\alpha^{\mathrm{II}}(\omega)$ and $\alpha^{\mathrm{III}}(\omega)$ can be evaluated by using the relation 22.13 .8 and 22.13 .9 in [1]:

$$
\begin{aligned}
\left\langle\alpha^{\mathrm{II}}, p_{i}\right\rangle_{\rho} & = \begin{cases}\frac{(-1)^{j} \Gamma\left(j-\frac{1}{2}\right)}{4 \Gamma\left(-\frac{1}{2}\right) \Gamma(j+2)}, & \text { if } i=2 j, \\
\frac{1}{6}, & \text { if } i=1, \\
0, & \text { if } i=2 j+1, i>1\end{cases} \\
\left\langle\alpha^{\mathrm{III}}, p_{i}\right\rangle_{\rho} & = \begin{cases}\frac{(-1)^{j} \Gamma\left(j-\frac{1}{4}\right) \Gamma\left(\frac{3}{4}\right)}{4 \Gamma\left(-\frac{1}{4}\right) \Gamma\left(j+\frac{7}{4}\right)}, & \text { if } i=2 j, \\
\frac{(-1)^{j} \Gamma\left(j+\frac{1}{4}\right) \Gamma\left(\frac{5}{4}\right)}{4 \Gamma\left(\frac{1}{4}\right) \Gamma\left(j+\frac{9}{4}\right)}, & \text { if } i=2 j+1,\end{cases}
\end{aligned}
$$


where $\Gamma(\cdot)$ denotes the Gamma function. The two last inner product are, in fact, rational numbers which can be computed without any error via a symbolic software. 